\title{
Clarify: Software for Interpreting and Presenting Statistical Results
}

\author{
Michael Tomz \\ Jason Wittenberg \\ Gary King ${ }^{1}$
}

January 5, 2003

\footnotetext{
${ }^{1}$ Tomz: Department of Political Science, Stanford University, Encina Hall, Stanford, CA 94305-6044, email tomz@stanford.edu; Wittenberg: Department of Political Science, University of Wisconsin, Madison, 1050 Bascom Mall, 221 North Hall, Madison, WI, 53706, email witty@polisci.wisc.edu; King: Center for Basic Research in the Social Sciences, 34 Kirkland Street, Harvard University, Cambridge MA 02138, email King@Harvard.Edu, website http://GKing.Harvard.Edu. Clarify is copyrighted, but you may copy and distribute this program provided that no charge is made and the copy is identical to the original. To request an exception, please contact Michael Tomz.
} 


\section{Contents}

1 Introduction $\quad 3$

2 Software requirements $\quad 3$

3 How to Install Clarify For the First Time $\quad 3$

3.1 Installing on Computers that are Connected to the Internet . . . . . . . . . . . 3

3.2 Installing on Computers that are Not Connected to the Internet $\ldots . . . . . . . .4$

4 How to update Clarify $\quad 5$

4.1 Updating from Version 2.0 or later $\ldots \ldots \ldots \ldots \ldots$

4.2 Updating from Version $1.2 \mathrm{x}$ or earlier $\ldots \ldots \ldots \ldots \ldots$

5 What Clarify Does $\quad 5$

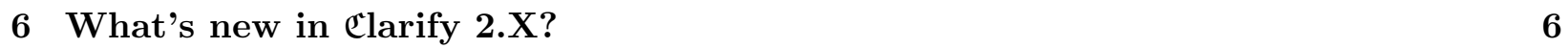

$\begin{array}{lll}7 & \text { A Simple Example } & 7\end{array}$

8 The Main Commands $r$

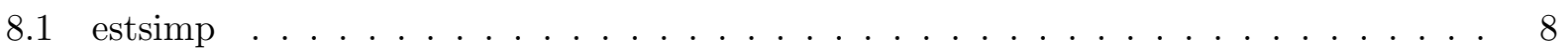

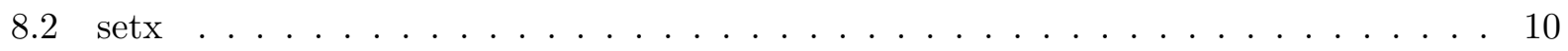

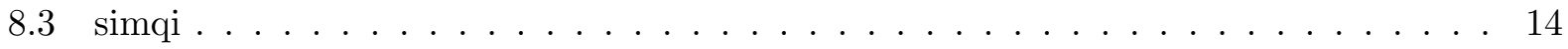

9 Frequently Asked Questions $\quad 19$

10 User-Contributed Modules $\quad 23$

11 Formulae - A Peek Under the Hood $\quad 24$

11.1 Algorithms for estsimp . . . . . . . . . . . . . . . . . . 24 


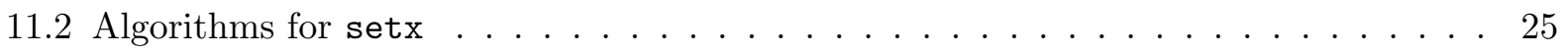

11.3 Algorithms for simqi . . . . . . . . . . . . . . . 26

12 References

13 Acknowledgements 


\section{Introduction}

Clarify is a program that uses Monte Carlo simulation to convert the raw output of statistical procedures into results that are of direct interest to researchers, without changing statistical assumptions or requiring new statistical models. The program, designed for use with the Stata statistics package, offers a convenient way to implement the techniques described in:

Gary King, Michael Tomz, and Jason Wittenberg (2000). "Making the Most of Statistical Analyses: Improving Interpretation and Presentation." American Journal of Political Science 44, no. 2 (April 2000): 347-61.

We recommend that you read this article before using the software.

Clarify simulates quantities of interest for the most commonly used statistical models, including linear regression, binary logit, binary probit, ordered logit, ordered probit, multinomial logit, Poisson regression, negative binomial regression, weibull regression, seemingly unrelated regression equations, and the additive logistic normal model for compositional data. Clarify Version 2.1 is forthcoming (2003) in Journal of Statistical Software.

\section{Software requirements}

Clarify works in conjunction with Stata Statistical Software, produced by Stata Corporation. Clarify will run on any platform (Windows, Unix, or Macintosh) where Stata is installed. You must have Stata 6.0 or later to run Clarify. To obtain a copy of Stata or learn more about the software, visit http://www.stata.com or send email to stata@stata.com.

\section{How to Install Clarify For the First Time}

If you do not have a previous version of Clarify on your personal computer or your network, there are two ways to install the software.

\subsection{Installing on Computers that are Connected to the Internet}

To install Clarify 2.1 for use with a personal copy of Stata, launch Stata and then type:

net from http://gking.harvard.edu/clarify/

net install clarify 
To install Clarify for use with a networked copy of Stata, launch Stata and then type:

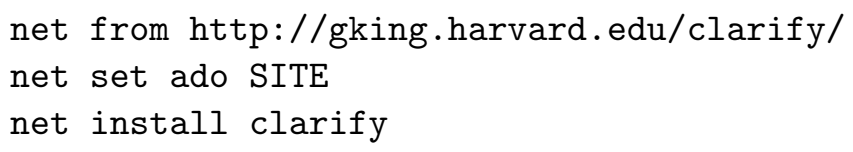

In either case, the following files will be installed on your computer: estsimp.ado, estsimp.hlp, setx.ado, setx.hlp, simqi.ado, simqi.hlp, sumqi.ado, sumqi.hlp, tlogit.ado, tlogit.hlp. Note that these files will be installed onto your adopath, the path where Stata searches for the files it needs. If you ever want to remove these files, simply type ado uninstall clarify

\subsection{Installing on Computers that are Not Connected to the Internet}

Download clarify.zip from http://gking.harvard.edu and copy that file to a floppy disk. Then insert the floppy into the disk drive of the machine that is not connected to the internet. Copy clarify.zip into a temporary directory or folder on the hard disk, and use a utility such as pkunzip (available for the PC at http://www.pkware.com) or StuffIt (available for the MacIntosh at http://www.aladdinsys.com/expander/index.html) to extract the files into your temporary directory. Finally, launch Stata and type the following commands from within Stata:

net from <temporary path designator>

net install clarify

Here, replace < temporary path designator $>$ with the path to the temporary directory or folder where you extracted the contents of clarify.zip. Thus, for example, if the archive were copied and opened into the c: \temp folder on a Windows machine, the appropriate installation commands would be:

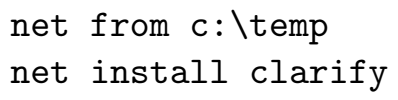

On a Macintosh, if you copied clarify.zip into a temporary folder called TEMPFOLDER, you could install the program by typing:

net from :TEMPFOLDER

net install clarify 


\section{How to update Clarify}

There are two ways to update $\mathfrak{C}$ larify. The choice depends on which version you currently have on your computer or network.

\subsection{Updating from Version 2.0 or later}

Once you've installed version 2.0 or later on your computer, it should be easy to update the program as new releases become available. If you have a personal copy of Stata, launch Stata and then type:

net from http://gking.harvard.edu/clarify/

net install clarify, replace

To update the copy on a network, launch Stata and then type:

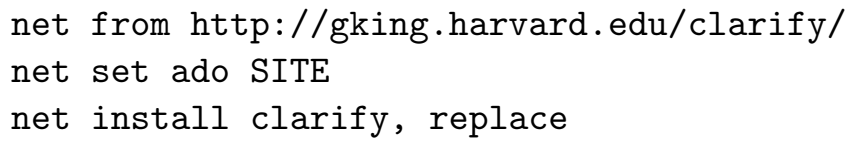

\subsection{Updating from Version 1.2x or earlier}

If you are using a version of Clarify released before June 2001, manually delete the following files from your Stata adopath, working directory, or network: estsimp.ado, estsimp.hlp, setx.ado, setx.hlp, simqi.ado, simqi.hlp, sumqi.ado, sumqi.hlp. Once you have done this, you may install Clarify according to the directions in Section 3.

\section{$5 \quad$ What Clarify Does}

Clarify uses stochastic simulation techniques to help researchers interpret and present their statistical results. It uses whatever statistical model you have chosen and, as such, changes no statistical assumptions. As a first step, the program draws simulations of the main and ancillary parameters $(\tilde{\gamma})$ from their asymptotic sampling distribution, in most cases a multivariate normal with mean equal to the vector of parameter estimates $(\hat{\gamma})$ and variance equal to the variance-covariance matrix of estimates $\hat{\mathrm{V}}(\hat{\gamma}){ }^{1}$ Thus,

$$
\tilde{\gamma} \sim \mathrm{N}(\hat{\gamma}, \hat{\mathrm{V}}(\hat{\gamma}))
$$

\footnotetext{
${ }^{1}$ There are two exceptions: the variance parameters for sureg, drawn from an inverse Wishart distribution; and the variance for regress, drawn from an inverse $\chi^{2}$ distribution. For details, see section 11 .
} 
By default the program draws $M=1000$ sets of simulated parameters, which should be sufficient for most applications.

Next, Clarify converts the simulated parameters into substantively interesting quantities, such as predicted values, expected values, or first differences. The user need only choose real or hypothetical values for the explanatory variables (the $X$ 's) and indicate which quantities should be calculated, conditional on those $X$ 's. The program allows researchers to calculate virtually any quantity that would shed light on a particular problem.

Clarify 2.1 simulates quantities of interest for the most commonly used statistical models, including linear regression, binary logit, binary probit, ordered logit, ordered probit, multinomial logit, Poisson regression, negative binomial regression, weibull regression, seemingly unrelated regression equations, and compositional data.

\section{$6 \quad$ What's new in Clarify 2.X?}

Clarify 2.0 includes a number of enhancements over previous versions, including:

- Support for more models, including weibull regression, seemingly unrelated regression equations, and the additive logistic normal model for compositional data.

- The ability to apply standard transformations — such as natural logs and exponents - to dependent variables, estimate a model, and then reverse those transformations when interpreting the results.

- Compatibility with Amelia. If you use multiple imputation to correct for problems with missing values, Clarify will analyze all the multiply imputed data sets, appropriately combine the results, and compute your quantity of interest automatically. (For information on the software program Amelia, see http://GKing.Harvard.Edu).

- An option to generate antithetical simulations, which guarantees that the mean of the simulated parameters equals the vector of point estimates, $\hat{\gamma}$, and usually reduces Monte Carlo variance.

- More powerful commands for setting the values of the explanatory variables (the $X^{\prime}$ 's), using either single or multiply-imputed datasets to compute descriptive statistics.

- The ability to re-display the previous point estimates by entering the estsimp command without any arguments.

- The option in the estsimp command to drop previously simulated parameters. 
Clarify 2.1 corrects two bugs in estsimp. The first concerns the simulation of parameters for the mlogit, nbreg, and sureg models. The second concerns the use of regress on multiply imputed datasets.

For automatic notifications (via email) of updates to Clarify, or to subscribe to the Clarify listserv, see http://GKing.Harvard.Edu/stats.shtml.

\section{$7 \quad$ A Simple Example}

Clarify is based on three simple commands:

estsimp (estimates the model and simulates its parameters)

setx (sets values of Xs before simulating quantities of interest)

simqi (simulates quantities of interest)

In general, the commands should be run in that order, although it is often useful to call setx and simqi many times after running estsimp. Clarify also contains two minor commands: sumqi and tlogit. sumqi assists in summarizing quantities of interest that have been saved to the dataset. tlogit applies the logistic transformation to one or more variables. For instructions on using these commands, consult the on-line help file. All Clarify commands can be run interactively at the Stata command line or in batch mode by using a Stata do-file.

Each command contains many options, which we describe in Section 8 of this manual. Here, we offer a simple example designed to show how Clarify can be used. With a dataset loaded into memory, type the following 3 commands:

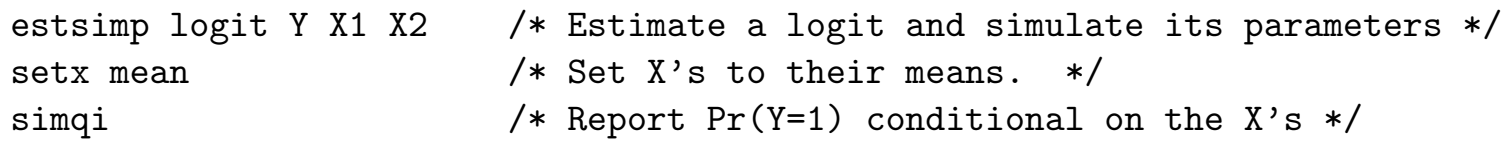

Each of these commands performs a particular operation, which we summarize here. A more detailed discussion appears in Section 8.

estsimp logit Y X1 X2 Clarify works by capturing and interpreting the statistical results that Stata produces when estimating a particular model. To use Clarify, insert the word estsimp at the beginning of an estimation command that you would normally run in Stata. In this example, the built-in Stata command is logit, the binary dependent variable is $Y$, and the explanatory variables are $X 1, X 2$, and a constant. Unless the user specifies otherwise, estsimp will save the simulated parameters as new variables bearing the names $b 1, b 2$, and $b 3$, which will hold simulations of the coefficients on $X 1, X 2$, as well as the constant term. 
If any of the variables $b 1$ through $b 3$ already exist in the dataset, Clarify will ask the user to delete those variables or choose different names for the simulations.

setx mean The setx command allows the user to choose a real or hypothetical value for each explanatory variable before computing quantities of interest. In this example, the command setx mean sets each $X$ equal to its average value.

simqi The simqi command computes and reports quantities of interest and associated measures of uncertainty. Used without specifying any options (many options are possible - see Section 8.3), simqi will compute intelligent default quantities that are appropriate for the model being estimated. In the case of logit, simqi will report the probability that $Y=1$.

\section{The Main Commands}

This section provides more detailed information about each of the main commands in Clarify: estsimp, setx, and simqi. Much of the information also appears in on-line help files, which can be viewed by searching the Stata help menu or entering help command name at the Stata prompt.

\section{1 estsimp}

\section{Format:}

estsimp modelname depvar [indepvars] [weight] [if exp] [in range]

[, sims(m) genname(newvar) antisim mi(file1 file2 ... filek) iout dropsims]

\section{Description:}

estsimp estimates a variety of statistical models and generates $M$ simulations of each parameter. Currently supported models include regress, logit, probit, ologit, oprobit, mlogit, poisson, nbreg. weibull, sureg, and the additive normal model for compositional data. The simulations are stored in new variables bearing the names newvar 1 , newvar $2, \ldots$, newvark, where $k$ is the number of parameters. Each variable has $M$ observations corresponding to the $M$ simulations. estsimp labels the simulated variables and lists their names on the screen, so you can verify what was simulated. The estsimp command accepts nearly all options that are typically available for the supported models. It also accepts several special options that are described below.

\section{Options:}

$\operatorname{sims}(\mathrm{M})$ specifies the number of simulations, $M$, which must be a positive integer. The default is 1000 simulations. If you choose a large number of simulations, you may need to allocate 
more memory to Stata. See $[R]$ memory in the Stata reference manual for more details about memory allocation.

genname(newvar) specifies a stub-name for the newly generated variables. If no stub is given, Stata will generate the variables $b 1, b 2, \ldots, b k$, otherwise it will generate newvar 1, newvar $2, \ldots$, newvark, provided that the variables do not exist in memory already.

antisim instructs estsimp to use antithetical simulations, in which numbers are drawn in pairs from the uniform $[0,1]$ distribution, with the second draw being one minus the first. The antithetical draws are then transformed to obtain simulations from a multivariate normal distribution. This procedure ensures that the mean of the simulations for a particular parameter is equal to the point estimate of that parameter even with a small number of simulations.

mi(filelist) allows estsimp to analyze multiply-imputed datasets: files in which missing values have been multiply imputed, such as created by Amelia. Enter the name for each imputed dataset you want to use, such as mi(file1 file2 file3). Alternatively, you can enter a common stub name for all imputed datasets, such as mi (file). In this case, estsimp assumes that you want to use all files in the working directory that are part of the uninterrupted sequence file1, file2, file3... estsimp will estimate the parameters for each dataset and use the estimates to generate simulations, which will reflect not only estimation uncertainty but also the uncertainty arising from the imputation process. Note: if the data in memory have been changed, you cannot specify the mi() option until you clear the memory or save the altered dataset. See the Amelia manual for more information about multiple imputation and the number of imputed files you need.

iout instructs estsimp to print intermediate output (a table of parameter estimates) for each imputed dataset that it analyzes. By default, estsimp suppresses the intermediate output and displays only the final estimates produced by combining the results from each imputed dataset.

dropsims drops the simulated parameters from the previous call to estsimp.

\section{Examples:}

To estimate a linear regression of $y$ on $x 1, x 2, x 3$, and a constant term; simulate 1000 sets of parameter estimates; and then save the simulations as $b 1, b 2, \ldots, b k$, type:

- estsimp regress y $\mathrm{x} 1 \mathrm{x} 2 \mathrm{x} 3$

In this example, Stata creates five new variables. The variables $b 1, b 2$ and $b 3$ will contain simulated coefficients for $x 1, x 2$ and $x 3$; $b 4$ will hold simulations of the constant term; and $b 5$ will contain simulated values for sigma squared, the mean squared error of the regression.

To simulate 500 sets of parameters from a logit regression and save the results as variables beginning with the letter "s", type: 
. estsimp logit y $\mathrm{x} 1 \mathrm{x} 2 \mathrm{x} 3, \operatorname{sims}(500)$ genname(s)

Since the logit model contains no ancillary parameters, this command will generate four new variables: $s 1, s 2, s 3$, and $s 4$. Variables $s 1-s 3$ are simulated coefficients for $x 1, x 2$ and $x 3$, and the final variable, $s 4$, is the simulated constant term.

To simulate 1000 sets of parameters from an ordered probit regression in which the dependent variable can assume three values (low, medium, and high), type:

- estsimp oprobit $\mathrm{y} \times 1 \mathrm{x} 2 \mathrm{x} 3$

The ordered probit model does not contain a constant term, but it does have ancillary parameters called cut-points. Thus, the estsimp command listed above generates five new variables. The variables $b 1, b 2$ and $b 3$ hold simulated coefficients for $x 1 x 2$ and $x 3$. Variables $b 4$ and $b 5$ contain simulations for the two cutpoints (cut1 and cut2).

To obtain antithetical variates, use the antisim option, as in

- estsimp oprobit $\mathrm{y} \times 1 \times 2 \times 3$, antisim

Suppose that we have three imputed datasets, called imp1.dta, imp2.dta, and imp3.dta. We could analyze all three datasets and combine the results by issuing the following command:

- estsimp oprobit $\mathrm{y} x 1 \mathrm{x} 2 \mathrm{x} 3$, mi(imp1 imp2 imp3)

The resulting simulations of the main and ancillary parameters would reflect both estimation uncertainty and the variability associated with the multiple imputations.

To view the intermediate output from each ordered probit estimation, add the iout option to the previous command, as in

- estsimp oprobit $\mathrm{y} \times 1 \mathrm{x} 2 \mathrm{x} 3$, mi(imp1 imp2 imp3) iout

\section{2 setx}

\section{Format:}

$\operatorname{set} x$ 


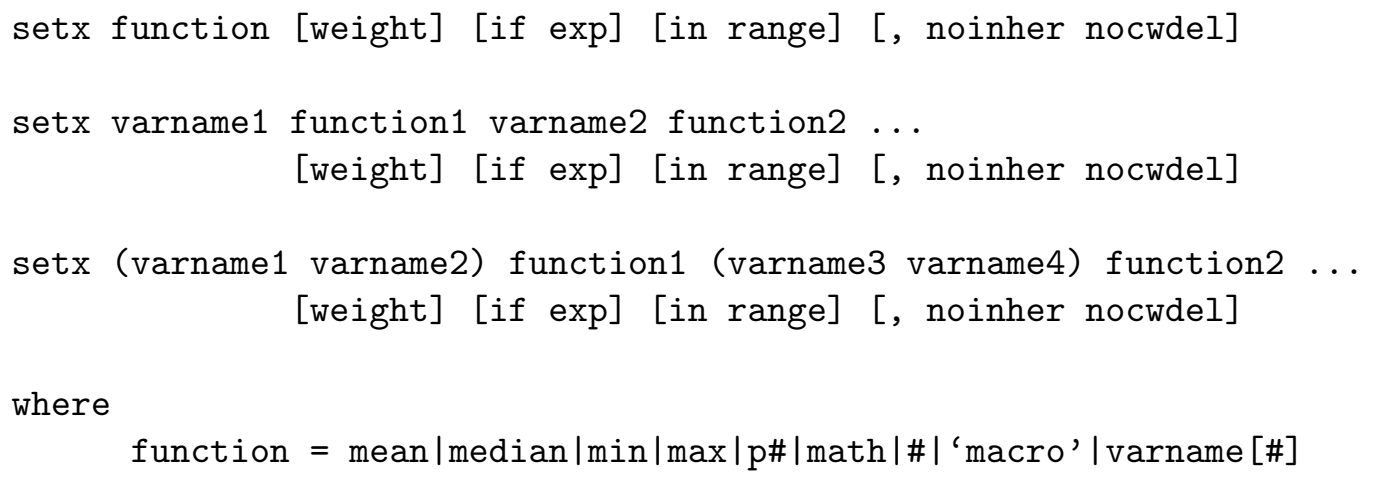

\section{Description:}

After simulating parameters from the last estimation (see Section 8.1), use setx to set values for the explanatory variables (the $X$ 's), change values that have already been set, or list the values that have been chosen. The main value types are

$\begin{array}{ll}\text { mean } & \text { arithmetic mean } \\ \text { median } & \text { median } \\ \text { min } & \text { minimum } \\ \text { max } & \text { maximum } \\ \text { p\# } & \text { \#th percentile } \\ \text { math } & \text { a mathematical expression, such as } 5 * 5 \text { or } \operatorname{sqrt}(23) \\ \# & \text { a numeric value, such as } 5 \\ \text { 'macro' } & \text { the contents of a local macro } \\ {[\#]} & \text { the value in the \#th observation of the dataset }\end{array}$

If you used multiply imputed datasets at the estimation stage, setx will use those same imputed datasets to calculate values for the explanatory variables. For instance, setx $\mathrm{x} 1$ mean would calculate the mean of $\mathrm{x} 1$ across all the imputed datasets.

When using setx or any other Stata command to calculate summary statistics such as means, medians, minimums, maximums, and percentiles, you must define the sample. At the estimation stage, Stata automatically disregards observations that do not satisfy the "if", "in", and "weight" conditions specified by the user. It also ignores observations with missing values on one or more variables. Before setting a particular variable equal to its mean or any other summary statistic, users must decide whether to calculate the statistic based only on observations that were used during the estimation stage, or to include other observations in the calculation.

By default, setx inherits the if-in-weight conditions from estsimp and disregards (casewise-deletes) any observation with missing values on the dependent or explanatory variables, unless you use the 
options for multiple imputation. You can specify different if-in-weight conditions by including them in the setx command line, and you can disregard all inherited conditions by using the noinher and nocwdel options described below.

The setx command is also used by another statistical package called relogit, which is also available from http://gking.harvard.edu. If you are running relogit with the wc() or pc() options, indicating that the data were selected on the dependent variable, setx will correct the selection bias when calculating summary statistics. For this reason, means and percentiles produced by setx may differ from means and percentiles of the (biased) sample. When the proportion of 1's in the population is known only to fall within a range, such as $\operatorname{pc}(.2 .3)$, setx will calculate bounds on the values of the explanatory variables. The result will be two $X$-vectors, the first assuming that the true proportion of 1's is at its lower bound, and the second conditional on the true proportion being at its upper bound. The program will pass these vectors to relogitq and use them to calculate bounds on quantities of interest. To set each explanatory variable at a single value that falls midway between its upper and lower bounds, use the nobound option that is described below.

setx accepts aweights and fweights. It also accepts the special options listed below.

Notes: (1) Setx will not accept spaces in mathematical expressions unless you enclose the expression in parentheses. setx $\mathrm{x} 4 \ln (20)$ is a valid command, but setx $\mathrm{x} 4 \ln (20)$ is a syntax error. Similarly, setx $\mathrm{x} 45 * 5$ and setx $\mathrm{x} 4(5 * 5)$ are valid, but setx $\mathrm{x} 45 * 5$ is not. (2) You may use square brackets ([]) when referring to observation numbers, e.g. setx $x$ [15], but do not use square brackets in mathematical expressions, or you may get unexpected results. We recommend that users check the values they have set with setx by entering setx without any arguments. (3) setx relies on three globals: the matrix mrt_xc and the macros mrt_vt and mrt_seto. If you change the values of these globals, the program may not work properly.

\section{Options:}

noinher causes setx to ignore all if-in-weight conditions that are inherited from estsimp. The user can specify new if-in-weight conditions by typing them as part of the setx command.

nocwdel forces setx to calculate summary statistics based on all valid observations for a given variable, even if the observations contain missing values for the other variables. If nocwdel is not specified, setx will casewise-delete observations with missing values.

nobound This option is available only after relogit, and only when the true proportion of 1's is assumed to fall within a specified range. Suppose the user typed $\operatorname{pc}(.2 .4)$ with relogit and then entered setx $\mathrm{x} 1$ mean. By default, setx would set the variable $\mathrm{x} 1$ equal to two values: the mean of $\mathrm{x} 1$, assuming that the true proportion of ones is only 0.2 , and the mean of $\mathrm{x} 1$, allowing that the true proportion is as high as 0.4 . Both values for $\mathrm{x} 1$ will be passed to relogitq and used to calculate bounds on quantities of interest. The nobound option overrides this procedure by setting each $\mathrm{x}$ to a single value: the midpoint of its upper and lower bound. Thus, the command setx $\mathrm{x} 1$ mean, nobound would set $\mathrm{x} 1$ equal to the following 
expression: $[(\operatorname{mean}(x 1) \mid \tau=0.2)+(\operatorname{mean}(x 1) \mid \tau=0.4)] / 2$, where $\tau$ represents the presumed proportion of 1 's in the population.

keepmrt is a programmer's option that instructs setx to return the matrix $r($ mrt_xc) without changing the globals mrt_xc, mrt_vt, and mrt_seto. If you don't understand what this means you should not use this option.

\section{Examples:}

To list values that have already been set:

- $\operatorname{set} x$

To set each explanatory variable at its mean:

. setx mean

To set each explanatory variable at its median, based on a sample in which $x 3>12$ and all inherited conditions are ignored and casewise deletion is suppressed:

- setx median if $x 3>12$, noinh nocw

To set each explanatory variable to the value contained in the 15 th observation of the dataset

$\operatorname{setx}[15]$

The command can also set each variable separately. For instance, to set $\mathrm{x} 1$ at its mean, $\mathrm{x} 2$ at its median, $\mathrm{x} 3$ at its minimum, $\mathrm{x} 4$ at its maximum, $\mathrm{x} 5$ at its 25 th percentile, $\mathrm{x} 6$ at $\ln (20)$, $\mathrm{x} 7$ at 2.5 , and $\mathrm{x} 8$ equal to a local macro called myval, type:

. $\operatorname{set} x \mathrm{x} 1$ mean $\mathrm{x} 2$ median $\mathrm{x} 3$ min $\mathrm{x} 4 \max \mathrm{x} 5 \mathrm{p} 25 \mathrm{x} 6 \ln (20) \mathrm{x} 72.5 \mathrm{x} 8$ 'myval'

To change the value of $\mathrm{x} 3$ from its previously chosen value to $5^{*} 5$

. $\operatorname{set} x \times 3 * 5$

To set all variables except x10 at their means, and fix $x 10$ at its 25 th percentile, call setx twice: once to set all variables at their means, and a second time to change the value of $x 10$ to its 25 th percentile. 
- setx mean

. $\operatorname{setx} \mathrm{x} 10 \mathrm{p} 25$

setx can also set values for groups of variables. To set $\mathrm{x} 1$ and $\mathrm{x} 2$ to their means, $\mathrm{x} 3$ to its median, and $\mathrm{x} 4$ and $\mathrm{x} 5$ to their 25 th percentiles, type:

. $\operatorname{setx}(\mathrm{x} 1 \mathrm{x} 2)$ mean $\mathrm{x} 3$ median $(\mathrm{x} 4 \mathrm{x} 5)$ p25

\section{3 simqi}

\section{Format:}

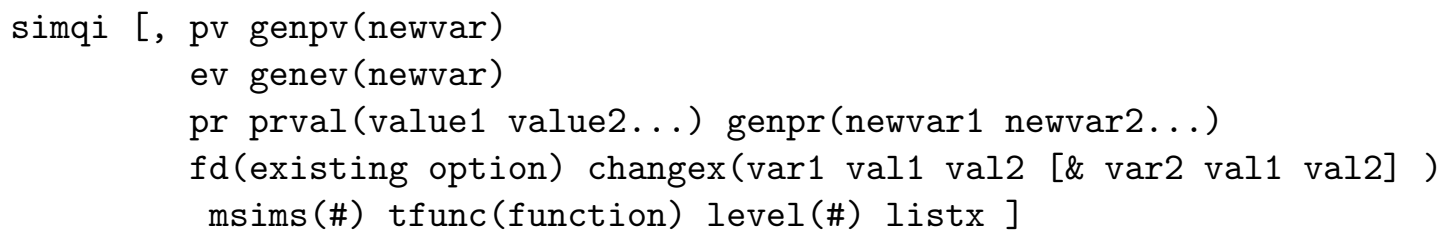

\section{Description:}

After simulating parameters from the last estimation (see Section 8.1) and setting values for the explanatory variables (see Section 8.2), use simqi to simulate various quantities of interest, including predicted values, expected values, and first differences.

Predicted values contain two forms of uncertainty: "fundamental" uncertainty arising from sheer randomness in the world, and "estimation" uncertainty caused by not having an infinite number of observations. More technically, predicted values are random draws of the dependent variable from the stochastic component of the statistical model, given a random draw from the posterior distribution of the unknown parameters.

If there were no estimation uncertainty, the expected value would be a single number representing the mean of the distribution of predicted values. But estimates are never certain, so the expected value must be a distribution rather than a point. To obtain this distribution, we average-away the fundamental variability, leaving only estimation uncertainty. For this reason, expected values have a smaller variance than predicted values, even though the point estimate should be roughly the same in both cases. simqi calculates two kinds of expected values, depending on the model: the expected value of $Y$, and the probability that $Y$ takes on a particular value. For models in which these two quantities are equal, simqi avoids redundancy by reporting only the probabilities.

Simulated expected values are equivalent to simulated probabilities for all the discrete choice models that simqi supports (logit, probit, ologit, oprobit, mlogit). In these models, the expected 
value of $Y$ is a vector, with each element indicating the probability that $Y=j$. Consider an ordered probit with outcomes 1, 2, 3. The expected value is $[\operatorname{Pr}(Y=1), \operatorname{Pr}(Y=2), \operatorname{Pr}(Y=3)]$, the mean of a multinomial distribution that generates the dependent variable.

A first difference is the difference between two expected values. To simulate first differences use the fd "wrapper", which is described below.

simqi can generate predicted values, expected values and first differences for all the models that it supports. By default, however, it will only report the quantities of interest that appear in the table below. To view other quantities of interest or save the simulated quantities as new variables that can be analyzed and graphed, use one of simqi's options.

\begin{tabular}{cc}
$\begin{array}{c}\text { Statistical } \\
\text { Model }\end{array}$ & $\begin{array}{c}\text { Quantities displayed } \\
\text { by default }\end{array}$ \\
\hline regress & $\mathrm{E}(\mathrm{Y})$ \\
logit & $\operatorname{Pr}(\mathrm{Y}=1)$ \\
probit & $\operatorname{Pr}(\mathrm{Y}=1)$ \\
ologit & $\operatorname{Pr}(\mathrm{Y}=\mathrm{j})$ for all j \\
oprobit & $\operatorname{Pr}(\mathrm{Y}=\mathrm{j})$ for all $\mathrm{j}$ \\
mlogit & $\operatorname{Pr}(\mathrm{Y}=\mathrm{j})$ for all $\mathrm{j}$ \\
poisson & $\mathrm{E}(\mathrm{Y})$ \\
nbreg & $\mathrm{E}(\mathrm{Y})$ \\
sureg & $\mathrm{E}\left(Y_{j}\right)$ for all equations j \\
weibull & $\mathrm{E}(\mathrm{Y})$ \\
\hline
\end{tabular}

\section{Options:}

pv displays a summary of the predicted values that simqi generated via simulation.

genpv(newvar) saves the predicted values as a new variable in the current dataset. Each "observation" of newvar represents one simulated predicted value.

pr displays a summary of the probabilities that simqi generated via simulation.

prval(value1 value $2 \ldots$...) instructs simqi to evaluate the probability that the dependent variable takes-on each of the listed values

genpr(newvar1 newvar2 ...) saves the simulated probabilities as new variables in the current dataset. Each new "observation" represents one simulated probability. If both the prval() option and the genpr() option are used, simqi will save $\operatorname{Pr}(\mathrm{Y}=$ value1) in newvar $1, \operatorname{Pr}(\mathrm{Y}=$ value2) in newvar2, etc. If the prval() option is not specified, genpr() will save the probabilities in the order that they appear on the screen. 
ev displays a summary of expected values that simqi generated via simulation. This option is not available for discrete choice models, where it is redundant with pr.

genev(newvar) saves the expected values in a new variable called newvar. Each observation of newvar represents one simulated expected value. This option is not available for discrete choice models, where it is redundant with genpr().

fd(existing option) is a wrapper that makes it easy to simulate first differences. Simply wrap the $\mathrm{fd}()$ wrapper around an existing option and specify the changex() option.

changex(var1 val1 val2) specifies how the explanatory variables (the $x$ 's) should change when evaluating a first difference. changex uses the same basic syntax as setx, except that each explanatory variable has two values: a starting value and an ending value. For instance, $\mathrm{fd}(\mathrm{ev})$ changex(x1 .2 .8) instructs simqi to simulate a change in the expected value of $Y$ caused by increasing $x 1$ from its starting value, 0.2 , to its ending value, to 0.8 .

level(\#) specifies the confidence level, in percent, for confidence intervals. The default is level(95) or the value set by set level. For more information on the set level command, see the on-line help for level.

msims(\#) sets the number of simulations to be used when calculating expected values. The number must be a positive integer. By default, the value of msims is set at 1000. simqi disregards the msims option whenever the expected value is parametrically defined.

listx instructs simqi to list the $\mathrm{x}$-values that were used to produce the quantities of interest. These values were set using the setx command.

tfunc(function) allows the user to specify a transformation function for transforming the dependent variable. This option is only available for regress and sureg. The currently supported functions are

\begin{tabular}{cl} 
Function & Transformation (for all variables j) \\
\hline squared & $y_{j} \longrightarrow y_{j} * y_{j}$ \\
sqrt & $\left.y_{j} \longrightarrow \sqrt{(} y_{j}\right)$ \\
$\exp$ & $y_{j} \longrightarrow e^{y_{j}}$ \\
In & $y_{j} \longrightarrow \ln \left(y_{j}\right)$ \\
logiti & $y_{j} \longrightarrow e^{y_{j}} /\left(1+\sum_{j} e^{y_{j}}\right)$ \\
\hline
\end{tabular}

\section{Basic Examples:}

To display the default quantities of interest for the last estimated model, type:

. simqi 
For a summary of the simulated expected values, type:

$$
\text { . simqi, ev }
$$

For a summary of the simulated probabilities, $\operatorname{Pr}(\mathrm{Y}=\mathrm{j})$, for all $\mathrm{j}$ categories of the dependent variable, type:

$$
\text { - simqi, pr }
$$

To display only a summary of $\operatorname{Pr}(\mathrm{Y}=1)$, the probability that the dependent variable takes on a value of 1 , type:

$$
\text { . simqi, prval(1) }
$$

To generate first differences, use the $\mathrm{fd}()$ wrapper and the changex () option. For instance, the following command will simulate the change in the expected value of $\mathrm{Y}$ caused by increasing $x 4$ from 3 to 7 , while holding other explanatory variables at their means

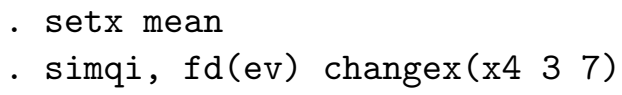

To simulate the change in the simulated probabilities, $\operatorname{Pr}(Y=j)$, for all $\mathrm{j}$ categories of the dependent variable, given an increase in $x 4$ from its minimum to its mean, type:

- setx mean

- simqi, $f d(p r)$ changex(x4 min mean)

If you are only interested in the change in $\operatorname{Pr}(\mathrm{Y}=1)$ caused by raising $\mathrm{x} 4$ from its 20 th to its 80 th percentile when other variables are held at their mean, type:

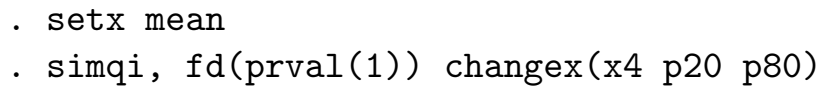

\section{More Intricate Examples:}

To display not only the simulated expected values but also the x-values used to produce them, we would type:

. simqi, ev listx 
simqi displays $95 \%$ confidence intervals by default, but we could modify the previous example to give a $90 \%$ confidence interval for the expected value:

. simqi, ev listx level(90)

To save the simulated expected values in a new variable called predval, type:

. simqi, genev(predval)

To simulate $\operatorname{Pr}(\mathrm{Y}=0), \operatorname{Pr}(\mathrm{Y}=3)$, and $\operatorname{Pr}(\mathrm{Y}=4)$, and then save the simulated probabilities as variables called simpr 0 , simpr3 and simpr4, type:

. simqi, prval( $\left(\begin{array}{lll}0 & 3\end{array}\right)$ genpr(simpr0 simpr3 simpr4)

The changex option can be arbitrarily complicated. Suppose that we want to simulate the change in $\operatorname{Pr}(\mathrm{Y}=1)$ caused by simultaneously increasing $x 1$ from .2 to .8 and $x 2$ from $\ln (7)$ to $\ln (10)$. The following lines will produce the quantities we seek:

- setx mean

. simqi, fd(prval(1)) changex(x1 .2 .8 x2 $\ln (7) \ln (10))$

We could augment the previous example by requesting a second first difference, caused by increasing $x 3$ from its median to its 90 th percentile. Simply separate the two changex requests with an ampersand.

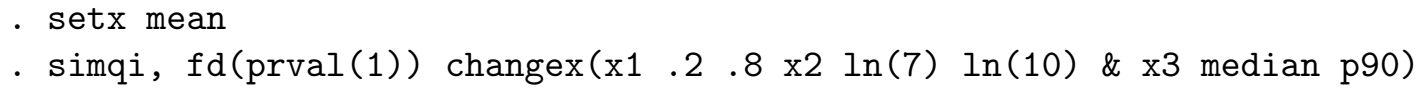

Likewise, the $\mathrm{fd}()$ option can be as intricate as we would like. For instance, suppose that we have run a poisson regression. We want to see what happens to $\operatorname{Pr}(Y=2), \operatorname{Pr}(Y=3)$, and the expected count when we increase $x 1$ from its minimum to its maximum. To obtain our quantities of interest, we would type:

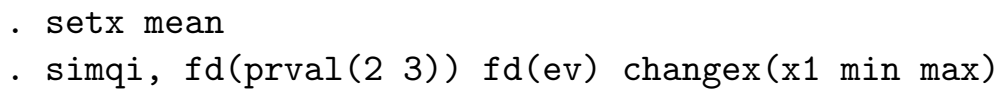

simqi allows us to save any simulated variable for subsequent analysis. To find the mean, standard deviation, and a confidence interval around any quantity of interest that has been saved in memory, use the sumqi command. To graph the simulations, use graph or kdensity. 
The tfunc() option reverses common transformations that users have applied to the dependent variable. Suppose that you have taken the log of the dependent variable before running estsimp regress. The command simqi would provide quantities of interest on the logged scale. If you wanted to reverse the transformation, thereby recovering the original scale, you could type

- simqi, tfunc(exp)

\section{$9 \quad$ Frequently Asked Questions}

Why does Clarify give slightly different results each time? Clarify uses random simulation to create quantities of interest and associated measures of uncertainty. Slight discrepancies are a result of taking a finite number of simulations and using a different random number seed. If you require more precision, increase the number of simulations drawn (see Section 8, Sub-section 8.1). If exactly the same numerical results are required, set the random number seed with the Stata command set seed before beginning the analysis.

Is it ok if some of my explanatory variables are statistically insignificant? Yes. Clarify computes quantities of interest based on all estimated coefficients, regardless of their level of statistical significance. This is not problematic because the true quantities of interest are usually the predicted values, expected values, and first differences, not the coefficients themselves. It is usually better to focus on the confidence intervals Clarify reports for each quantity it computes than the standard errors of coefficients. Moreover, even coefficients that are not statistically significant can provide important information: after all, a coefficient that is not significantly different from zero will probably significantly different from almost all other numbers.

How do I know how many simulations $(M)$ to draw? In our experience $M=1000$ is sufficient for most analyses. One check on the adequacy of $M$ is to verify that the means of the simulated parameters are equal to the estimated parameters within the desired degree of precision. If they are not, increase $M$ until you achieve the desired precision. A more general check is to do the same Clarify run twice; if you need three digits of precision to present in a table, then both runs should be the same to three digits. Otherwise increase $M$ and try again. Be aware that in larger models increasing $M$ may add noticably to the computer time and memory required for simulation. Using antithetical simulation can greatly reduce the needed number of simulations for some purposes.

How can I set the X's equal to the actual values in my dataset? You can use the setx command to set the x's at any value you'd like, including the actual values that appear in the 
How can I use Clarify to analyze compositional data? The procedure involves four basic steps:

1. Run tlogit to transform the vote shares (or other compositional data) into log ratios

2. Run estsimp sureg to estimate a seemingly unrelated regression and simulate the parameters

3. Run setx to choose real or hypothetical values for the explanatory variables $(X$ 's)

4. Run simqi with the tfunc() option to simulate the distribution of votes, conditional on the simulated parameters and chosen $X$ 's.

Suppose that we are studying a political system with 500 electoral districts. Each observation or row in the dataset pertains to one of those districts. In this example, we have three political parties that each garner a percentage of the vote. Their vote shares, collected in variables v1, v2, and v3, sum to 100 percent.

First, we select party 3 as our reference party and transform the vote shares of the other two parties into $\log$ ratios with respect to party 3 . Thus, $y 1=\ln (v 1 / v 3)$ and $y 2=\ln (v 2 / v 3)$. The appropriate syntax in Clarify is tlogit v1 y1 v2 y2, base(y3) percent, which will create two new variables: $\mathrm{y} 1$ and $\mathrm{y} 2$, which are the $\log$ ratios for $\mathrm{v} 1$ and $\mathrm{v} 2$ with respect to the base variable v3.

Second, use the estsimp command to run a seemingly unrelated regression model with the log ratios y1 and y2 as our dependent variables. The syntax is estsimp sureg ( $11 \mathrm{x} 1 \mathrm{x} 2)$ ( $\mathrm{y} 2 \mathrm{x} 3$ $\mathrm{x} 4)$. Each equation is enclosed in parentheses. Thus, the first equation states that the log ratio $\mathrm{y} 1$ is a linear function of the explanatory variables $\mathrm{x} 1$ and $\mathrm{x} 2$. The program will automatically add a constant term, as well, unless the user asks that it be suppressed. Likewise, the second equation states that $\mathrm{y} 2$ is a linear function of $\mathrm{x} 3, \mathrm{x} 4$, and a constant. The estsimp command will estimate the model and simulate the parameters. By default, estsimp will draw 1000 values for each parameter. In this example, the program would draw 1000 sets of betas (each set has six elements: three betas for equation 1 and three for equation two); the program would also generate 1000 simulations of $\Sigma$, a $2 \times 2$ matrix that governs the relationship between the errors of the two equations. Clarify will store these simulations in memory for subsequent use.

Third, use the setx command to choose some hypothetical or real values for our explanatory variables. For instance, type setx $(\mathrm{x} 1 \mathrm{x} 2)$ mean $\mathrm{x} 315 \mathrm{x} 4 \mathrm{p} 20$ to set variables $\mathrm{x} 1$ and $\mathrm{x} 2$ at their respective means, $\mathrm{x} 3$ equal to the number 15 , and $\mathrm{x} 4$ equal to its twentieth percentile.

Finally, use the simqi command to simulate quantities of interest, such as the predicted distribution of votes. The command is simqi, pv tfunc(logiti), where tfunc(logiti) tells the program to apply the inverse logistic function to transform the log ratios into shares of the total vote. 
How did you generate the graphs in your paper? Clarify does not automatically produce graphs. In order to produce a graph, you will need to use Stata's graphics commands. The sequence of commands used to generate Figure 1 from our paper is:

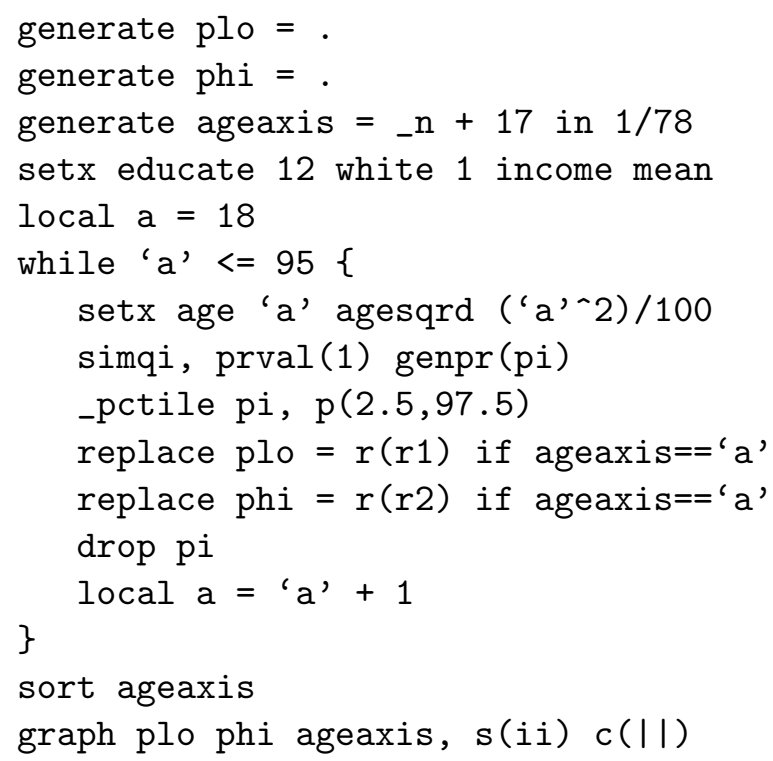

How can I create a log of all the commands and output in a Clarify session? See [R] $\log$ in the Stata reference manual or consult the on-line help for the log command.

How do I use imputed data sets from Amelia in Clarify? Amelia deals with missing data problems by filling in your missing values with imputed values. It creates several imputed data sets, where the observed data are identical and the missing data differ by the degree to which the correct values can be predicted from the observed data. When Amelia is finished, you have a small group (usually 4-5) data sets, or for some difficult problems with high imputation variance a larger number (see the Amelia manual for details). Clarify will automatically and appropriately combine the results from these data sets.

For example, suppose you have 2 imputed datasets called ds1 and ds2. Then follow these steps:

1. Load either ds1 or ds2 into memory by typing "use ds1"

2. Run a model that clarify supports and specify the mi() option. If ds1 and ds2 are in your working directory, the command might look something like this: estsimp regress y $\mathrm{x} 1 \mathrm{x} 2$, mi (ds1 ds2). Clarify will report the parameter estimates and standard errors, based on all imputed datasets named. 
3. To set the X's (or to get summary statistics for the imputed datasets), use the setx command. Setx will remember the locations of the imputed datasets that you specified at the estsimp stage. For example, "setx mean" would set x1 and x2 to their means, again based on all the imputed datasets. If you want to see actual numerical values of those means, type "setx" by itself (after running "setx mean") and clarify will list out the means for each x.

How do I cite this program? If you use this software, please cite

Michael Tomz, Jason Wittenberg, and Gary King. 2003. CLARIFY: Software for Interpreting and Presenting Statistical Results. Version 2.1. Stanford University, University of Wisconsin, and Harvard University. January 5. Available at http://gking.harvard.edu/

and

Gary King, Michael Tomz, and Jason Wittenberg (2000). "Making the Most of Statistical Analyses: Improving Interpretation and Presentation." American Journal of Political Science 44, no. 2 (April 2000): 347-61.

Can I share Clarify with others? Clarify is (C) Copyright, 1998-2003, Michael Tomz, Jason Wittenberg and Gary King, All Rights Reserved. You may copy and distribute this program provided the copy is identical to the original and you do not charge for it. To request an exception, please contact Michael Tomz, tomz@stanford.edu.

We recommend that you distribute the current version of this program, which is available from http://GKing. Harvard.Edu, or just give out the url.

What if I find a bug? First, get the most recent version (from http://gking.harvard.edu) and try to replicate the problem. If the problem persists, copy down exactly what you see on the screen when the program crashes, and email it along with the command you used to generate the error, to the Clarify Listserv at clarify@latte.harvard.edu.

You may also send comments by mail to Professor Michael Tomz, Department of Political Science, Encina Hall, Stanford University, Stanford, CA 94305-6044.

\section{User-Contributed Modules}

Fred Wolfe has contributed a module called "QSIM", which provides a wrapper automates simulation with dummy variables produced by Stata's xi prefix. For details about this and other 
user-contributed modules type net search clarify from the Stata command prompt.

\section{Formulae - A Peek Under the Hood}

This section is intended for advanced users who want details about the algorithms that Clarify uses to simulate parameters, set values for the explanatory variables, and compute quantities of interest. Other information is in our article. We welcome any suggestions for improvement.

\subsection{Algorithms for estsimp}

Recall that the estsimp command performs two functions: it estimates the main and ancillary parameters $(\gamma)$ of the statistical model, and it draws simulations of those parameters from their asymptotic sampling distribution.

Typically, the sampling distribution is multivariate normal with mean equal to the point-estimates of the parameters $(\hat{\gamma})$ and variance equal to the variance-covariance matrix of estimates $\hat{V}(\hat{\gamma})$. The current version of Clarify contains two exceptions to this rule.

In the case of linear regression, the effect coefficients $(\beta \mathrm{s})$ are drawn from a multivariate normal, but simulations of the (homoskedastic) variance $\sigma^{2}$ are obtained in a separate step from a scaled inverse $\chi^{2}$ distribution with $\nu=n-k$ degrees of freedom, where $n$ is the number of observations in the dataset and $k$ is the number of explanatory variables, including the constant term (see Gelman, et al., 1995, p. 237). The two-step procedure is legitimate because the effect coefficients and variance parameter are orthogonal in a linear regression; the procedure is desirable because $\sigma^{2}$ is strictly positive, and therefore more appropriately drawn from its exact posterior than from a normal distribution. To obtain simulations of $\sigma^{2}$, the program draws $c$ from a $\chi^{2}$ with $\nu$ degrees of freedom, and then calculates $\tilde{\sigma}^{2}=\nu \sigma^{2} / c$. The resulting draws have an expected value of $\left(\frac{\nu}{\nu-2}\right) \hat{\sigma}^{2}$, which approaches $\hat{\sigma}^{2}$ as $\nu \rightarrow \infty$.

Likewise, the effect coefficients $(\beta \mathrm{s})$ of a seemingly unrelated regression are drawn from the multivariate normal, but simulations of the variance matrix $\Sigma$ are obtained in a separate step. Here, the appropriate posterior distribution is the inverse Wishart (Gelman, et al., 1995, p. 481) with $\nu$ degrees of freedom and dimension $p$, where $p$ is the number of equations in the seemingly unrelated regression model. In cases where the number of explanatory varables varies from one equation to the next, Clarify calculates $n-k$ for each equation and sets $\nu$ equal to the mean of those values. To obtain simulations of $\Sigma$, the program draws from a Wishart with scale factor $(\nu \hat{\Sigma})^{-1}$ and inverts the draws. The algorithm for drawing from the Wishart relies on Bartlett's decomposition, which is concisely summarized in Johnson (1987, p. 204) and Ripley (1987, pp. 99-100). estsimp produces draws that have an expected value of $\frac{\nu}{\nu-p-1} \hat{\Sigma}$, which approaches $\hat{\Sigma}$ as $\nu$ goes to infinity. In small samples this procedure is conservative, since $\nu>\nu-p-1$, implying that $E(\tilde{\Sigma})>\hat{\Sigma}$. 
For all models, simulations of the main and ancillary parameters are random. This means that, in any given run of estsimp, the average value of $\tilde{\gamma}$ may be slightly smaller or larger than the point estimate $\hat{\gamma}$, though the approximation becomes more precise with a higher number of simulations. Users can force the mean of the simulated parameters to equal the vector of point estimates by requesting antithetical simulations (Stern 1997, pp. 2028-29). The antisim option instructs the program to draw random numbers in pairs from the uniform $[0,1]$ distribution, with the second draw being the complement of the first. For instance, if the first draw is 0.3 then the complementary draw is 0.7. The draws are, therefore, exactly balanced around the mean of the uniform distribution. These anthithetical simulations are then used to obtain antithetical or balanced draws from the multivariate normal via the inverse CDF method.

When users are analyzing a single dataset, Clarify estimates a single vector $\hat{\gamma}$ with variance $\hat{V}(\hat{\gamma})$ and draws all $M$ simulations based on those estimates. The table that appears on the screen gives the exact point estimates and standard errors, instead of reporting the means and standard deviations of the simulations.

The procedure is somewhat more complicated when the researcher employs the mi option to analyze several imputed datasets. In this case, estsimp repeats the following algorithm $I$ times, where $I$ is the number of completed datasets: estimate the parameters and their variance-covariance matrix conditional on the information in dataset $i(i=1,2, \ldots, I)$, and then draw $M / I$ sets of parameters from their sampling distribution. By repeating this algorithm $I$ times, the program generates $M$ sets of simulated parameters. The output table gives the analytical point-estimate, standard error, and $t$-statistic for each parameter, instead of reporting the means and standard deviations of the simulations. Specifically, the multiple-imputation point estimate for parameter $q$ is $\bar{q}=\frac{1}{I} \sum_{i=1}^{I} \hat{q}_{i}$ and the variance associated with $\bar{q}$ is a weighted combination of the within-imputation and betweenimputation variances: $V(\bar{q})=\bar{w}+\left(1+I^{-1}\right) b$, where $\bar{w}=\frac{1}{I} \sum_{i=1}^{I} V\left(\hat{q}_{i}\right)$ and $b=\frac{1}{I-1} \sum_{i=1}^{I}\left(\hat{q}_{i}-\bar{q}\right)^{2}$. The ratio of $\bar{q}$ (the parameter estimate) to $V(\bar{q})^{1 / 2}$ (its standard error) forms a $t$-statistic with degrees of freedom $\nu=(I-1)\left[1+\frac{\bar{u}}{\left(1+I^{-1}\right) b}\right]^{2}$. For more information about these procedures, see King, et al. (2001) and Schafer (1997, pp. 109-110).

\subsection{Algorithms for setx}

setx allows the user to choose real or hypothetical values for the explanatory variables (the $X \mathrm{~s}$ ). The program employs standard formulae for the mean, the minimum, the maximum, percentiles, and other descriptive statistics. If the user is analyzing several imputed datasets, setx will calculate the average statistic across the datasets. For instance, the command set $\mathrm{x} 1$ mean will calculate the mean of $\mathrm{x} 1$ in each dataset, and then set $\mathrm{x} 1$ equal to the average of those means. Similarly, the command setx $\mathrm{x} 1 \mathrm{x} 1$ [3] will obtain the value of $\mathrm{x} 1$ in the third row of each imputed dataset, and then set $\mathrm{x} 1$ equal to the average of those values. 


\subsection{Algorithms for simqi}

simqi simulates quantities of interest based on the parameters that were generated by estsimp and the $x$-values that were chosen with setx. The program obtains simulations of the dependent variable and uses them to calculate expected values, probabilities, first differences, and other quantities of interest. This procedure works in all cases but involves some approximation error, which users can make arbitrarily small by choosing a sufficient number of simulations. In many cases, though, shortcuts exist that can curtail both computation time and approximation error. simqi employs such shortcuts whenever possible. Here, we sketch the algorithms for each model that Clarify supports.

regress: The exact algorithm in simqi depends on whether the user has transformed the dependent variable (e.g., taken the log of $y$ ) prior to estimation. If no such transformation has occurred, the program generates one predicted value according to the formula $\tilde{y}=X_{c} \tilde{\beta}+\tilde{\epsilon}$, where $\tilde{\beta}$ is a vector of simulated effect coefficients and $\tilde{\epsilon}$ is one draw from $N\left(0, \tilde{\sigma}^{2}\right)$. Likewise, the program simulates one expected value as $\tilde{E}(y)=X_{c} \tilde{\beta}$. The algorithm becomes a bit more complicated if the user transformed the dependent variable prior to estimation, and would like to reverse the transformation when interpreting the results. Let $f$ represent a function, as identified by the tfunc () option, that reverses the transformation. If $f$ has been specified, the program simulates one predicted value according to the formula $f\left(X_{c} \tilde{\beta}+\tilde{\epsilon}\right)$. For an expected value, the program draws $m$ values of $\tilde{\epsilon}_{d}$ $(d=1,2, \ldots, m)$ from $N\left(0, \tilde{\sigma}^{2}\right)$ and then computes $(1 / m) \sum_{d=1}^{m} f\left(X_{c} \tilde{\beta}+\tilde{\epsilon}_{d}\right)$, which is the average of $m$ predicted values.

logit: The formula for $\tilde{\pi}$, the simulated probability that the dependent variable $y$ takes on a

value of 1 , is $1 /\left(1+e^{-X_{c} \tilde{\beta}}\right)$. To obtain one simulation of $y$, the program draws a number from the Bernoulli distribution with parameter $\tilde{\pi}$.

probit: The formula for $\tilde{\pi}$, the simulated probability that the dependent variable $y$ takes on a value of 1 , is $\Phi\left(X_{c} \tilde{\beta}\right)$ where $\Phi$ is the c.d.f. of the standard normal distribution. To obtain one simulation of $y$, the program draws a number from the Bernoulli distribution with parameter $\tilde{\pi}$.

ologit: The exact formula depends on the number of categories in the dependent variable. Suppose there are three categories. Let $\tilde{\beta}$ represent one simulated vector of effect coefficients and let $\tilde{\tau}_{l o}$ and $\tilde{\tau}_{h i}$ stand for draws of the cutpoints. To obtain one simulation of the probabilities for each category $(y=0, y=1, y=2)$, the program calculates: $\tilde{\pi}_{0} \equiv \tilde{\operatorname{Pr}}(y=0)=\frac{1}{1+e^{\left(X_{c} \tilde{\beta}-\tilde{\tau}_{l o}\right)}}, \tilde{\pi}_{1} \equiv \tilde{\operatorname{Pr}}(y=1)=$ $\frac{1}{1+e^{\left(X_{c} \tilde{\beta}-\tilde{\tau}_{h i}\right)}}-\frac{1}{1+e^{\left(X_{c} \tilde{\beta}-\tilde{\tau}_{l o}\right)}}$, and $\tilde{\pi}_{2} \equiv \tilde{\operatorname{Pr}}(y=2)=1-\frac{1}{1+e^{\left(X_{c} \tilde{\beta}-\tilde{\tau}_{h i}\right)}}$. With these results, the program can draw a predicted value, $\tilde{y}$, from a multinomial distribution with parameters $\tilde{\pi}_{0}, \tilde{\pi}_{1}$, $\tilde{\pi}_{2}$, and $n=1$.

oprobit: The exact formula depends on the number of categories in the dependent variable. Suppose there are three categories. Let $\tilde{\beta}$ represent one simulated vector of effect coefficients and let $\tilde{\tau}_{l o}$ and $\tilde{\tau}_{h i}$ stand for draws of the cutpoints. To obtain one simulation of the probabilities for 
each category $(y=0, y=1, y=2)$, the program calculates $\tilde{\pi}_{0} \equiv \tilde{\operatorname{Pr}}(y=0)=\Phi\left(\tilde{\tau}_{l o}-X_{c} \tilde{\beta}\right)$, $\tilde{\pi}_{1} \equiv \tilde{\operatorname{Pr}}(y=1)=\Phi\left(\tilde{\tau}_{h i}-X_{c} \tilde{\beta}\right)-\Phi\left(\tilde{\tau}_{l o}-X_{c} \tilde{\beta}\right)$, and $\tilde{\pi}_{2} \equiv \tilde{P r}(y=2)=\Phi\left(X_{c} \tilde{\beta}-\tilde{\tau}_{h i}\right)$. With these results, the program can draw a predicted value, $\tilde{y}$, from a multinomial distribution with parameters $\tilde{\pi}_{0}, \tilde{\pi}_{1}, \tilde{\pi}_{2}$, and $n=1$.

mlogit: The probability equation for the $K$ nominal outcomes of the multinomial $\operatorname{logit}$ is $\tilde{\pi}_{j} \equiv$ $\tilde{\operatorname{Pr}}(y=j)=\frac{e^{X_{c} \tilde{\beta}_{j}}}{\sum_{k=1}^{K} e^{X_{c} \tilde{\beta}_{k}}}$, where one of the $J$ outcomes is the base category, such that the effect coefficients $\tilde{\beta}$ for that category are set to zero. With these results, the program can draw a predicted value, $\tilde{y}$, from a multinomial distribution with parameters equal to the $\tilde{\pi}$ s and $n=1$.

poisson: The formula for the expected value $\tilde{\mu}$ is $e^{X_{c} \tilde{\beta}}$, and the probability that the dependent variable takes on the integer value $j$ is $\tilde{\operatorname{Pr}} r(y=j)=\frac{e^{-\tilde{\mu}} \tilde{\mu}^{j}}{j !}$. To obtain one predicted value, the program draws $\tilde{y}$ from a Poisson distribution with parameter $\tilde{\mu}$. The Poisson simulator is adapted from Press, et al. (1992), pp. 293-95.

nbreg: The formula for the expected value $\tilde{\mu}$ is $e^{X_{c} \tilde{\beta}}$, just as in the Poisson regression model (Long

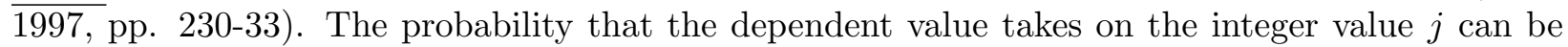
simulated as $\tilde{\operatorname{Pr}}(y=j)=\frac{\Gamma\left(j+\tilde{\alpha}^{-1}\right)}{j ! \Gamma\left(\tilde{\alpha}^{-1}\right)}\left(\frac{\tilde{\alpha}^{-1}}{\tilde{\alpha}^{-1}+\tilde{\mu}}\right)^{\tilde{\alpha}^{-1}}\left(\frac{\tilde{\mu}}{\tilde{\alpha}^{-1}+\tilde{\mu}}\right)^{j}$. simqi obtains $\tilde{\alpha}$, the "overdispersion" parameter, by drawing simulations of $\ln (\alpha)$ and the other parameters from the multivariate normal distribution and then calculating $e^{\ln (\alpha)}$. To obtain a predicted value $\tilde{y}$, the program draws one number from a poisson distribution with mean $e^{X_{c} \tilde{\beta}+\tilde{\epsilon}}$, where $e^{\tilde{\epsilon}}$ is simulated from a gamma distribution with shape parameter $\tilde{\alpha}^{-1}$ and scale parameter $\tilde{\alpha}$. When $\tilde{\alpha}^{-1}<1$, the gamma simulator is based on the algorithm developed by Ahrens and Dieter, as described in Ripley (1987, p. 88). For other values of $\tilde{\alpha}^{-1}$, the gamma simulator is based on the procedure by Best, as described in Devroye (1986, p. 410).

sureg: As with regress, the algorithm for interpreting the results of a sureg depends on whether the user transformed the dependent variable. If the user estimated the model without transforming the dependent variable, the program generates one predicted value for equation $k$ according to the formula $\tilde{y}_{k}=X_{c} \tilde{\beta}_{k}+\tilde{\epsilon}_{k}$, where $\tilde{\beta}_{k}$ is a vector of simulated effect coefficients for equation $k$ and $\tilde{\epsilon}_{k}$ is a simulated disturbance term for that equation. Disturbances for all equations are drawn simultaneously from a multivariate normal distribution with mean 0 and variance matrix $\tilde{\Sigma}$, as obtained from the inverse Wishart. Likewise, the program simulates one expected value for equation $k$ as $\tilde{E}\left(y_{k}\right)=X_{c} \tilde{\beta}_{k}$. If the user has transformed the dependent variable, let $f$ represent the function that reverses the transformation. The program simulates one predicted value for equation $k$ according to the formula $f\left(X_{c} \tilde{\beta}_{k}+\tilde{\epsilon}_{k}\right)$. For an expected value, the program draws $m$ sets of disturbance terms from $N(0, \tilde{\Sigma})$ and indexes them as $\tilde{\epsilon}_{k, d}$, where $k$ marks the equation and $d=1,2, \ldots, m$. Then, for each equation $k$ the program computes $(1 / m) \sum_{d=1}^{m} f\left(X_{c} \tilde{\beta}_{k}+\tilde{\epsilon}_{k, d}\right)$, which is the average of $m$ predicted values.

weibull: The algorithm depends on which metric, proportional hazard $(\mathrm{PH})$ or accelerated failuretime (AFT) metric, was used at the estsimp stage. The expected value is defined as $\tilde{\lambda}^{1 / \tilde{p}} \Gamma(1+1 / \tilde{p})$. 
In the AFT metric, $\tilde{\lambda}=e^{-X_{c} \tilde{\beta} \tilde{p}}$; in the PH metric, $\tilde{\lambda}=e^{X_{c} \tilde{\beta}}$. The program obtains simulations of the ancillary shape parameter $p$ drawing by $\ln (p)$ and the other parameters from a multivariate normal distribution and then calculating $e^{\ln (p)}$. To obtain a predicted value, the program draws one number from the Weibull distribution with parameters $\tilde{\lambda}$ and $\tilde{p}$. 


\section{References}

Devroye, Luc (1986). Non-Uniform Random Variate Generation. New York: Springer-Verlag.

King, Gary, Michael Tomz, and Jason Wittenberg (2000). "Making the Most of Statistical Analyses: Improving Interpretation and Presentation." American Journal of Political Science 44, no. 2 (April 2000): 347-61, copy at http://gking.harvard.edu/files/abs/making-abs.shtml.

King, Gary, James Honaker, Anne Joseph, and Kenneth Scheve (2001). "Analyzing Incomplete Political Science Data: An Alternative Algorithm for Multiple Imputation." American Political Science Review 95, no. 1 (March 2001): 49-69, copy at http://gking.harvard.edu/files/abs/ evil-abs.shtml.

Johnson, Mark E. (1987). Multivariate Statistical Simulation. New York: John Wiley \& Sons.

Long, J. Scott (1997). Regression Models for Categorical and Limited Dependent Variables. Thousand Oaks, CA: SAGE Publications.

Press, William H., Saul A. Teukolsky, William T. Vetterling, and Brian P. Flannery (1992). Numerical Recipes in C: The Art of Scientific Computing, 2nd ed. New York: Cambridge University Press.

Ripley, Brian D. (1987). Stochastic Simulation. New York: John Wiley \& Sons.

Schafer, J.L. (1997). Analysis of Incomplete Multivariate Data. New York: Chapman \& Hall.

Stern, Steven (1997). "Simulation-Based Estimation." Journal of Economic Literature 35, no. 4 (December): 2006-39.

\section{Acknowledgements}

We gratefully acknowledge comments and suggestions by Nick Cox, William Gould, Andrew D. Martin, Ken Scheve, Randy Stevenson, and the editors and anonymous reviews of the Journal of Statistical Software. We also wish to thank the many users of Clarify who have provided numerous suggestions for making the software more flexible and user-friendly. Parts of this program were inspired by J. Scott Long, "CATDEV: Stata Modules for Interpretation of Categorical Dependent Variables" (Indiana University, April 16, 1998). The multiple imputation procedure in estsimp extends the miest command written by Ken Scheve (Harvard University, February 6, 1999). 